\title{
ON BARE POINTS
}

\author{
S. J. BERNAU
}

(Received 7 February 1966)

This note shows that the set of bare points of a compact convex subset of a normed linear space is, in general, a proper subset of its set of exposed points.

We begin with some notation and standard definitions. All linear spaces are assumed real. Let $M$ be a subset of a real normed linear space $E$; $c l M$ and conv $M$ denote the closure and convex hull of $M$ respectively. If $M$ is convex an extreme point of $M$ is a point of $M$ which is not interior to any line segment contained in $M$; and an exposed point of $M$ is a point $p$ of $M$ such that for some closed supporting hyperplane $H, H \cap M=\{p\}$.

We write ext $M$ and $\exp M$ for the sets of extreme and exposed points of $M$. If $U$ is the unit ball of $E$ and $S$ the unit sphere

$$
(U=\{x \in E:\|x\| \leqq 1\}, S=\{x \in E:\|x\|=1\})
$$

then $U$ is rotund if $S=$ ext $U$ and smooth if at each point of $S$ there is only one supporting hyperplane of $U$ [3, VII $\S 2]$. We will say that the norm in $E$ is rotund or smooth if the unit ball has the corresponding property.

In [5] G. H. Orland defined a bare point of a compact convex plane set $A$ to be a point $p$ of $A$ such that there exists a closed disc containing $A$ and having $p$ on its circumference. Orland proves that $A=\operatorname{cl} \operatorname{conv} B$ where $B$ is the set of bare points of $A$. In [1] S. K. Berberian points out that Orland's definition and proof extend at once to inner product spaces and give there a proof of the Krein-Mil'man Theorem which does not require the use of Zorn's Lemma. (It is clear that bare points are extreme points in this context.)

The idea of a bare point of a bounded convex set is readily defined in any normed space. It is a point of the set on the boundary sphere of some closed ball containing the set. The notion is essentially a metric one. Changing to an equivalent norm will almost certainly change sets of bare points. In particular, if the unit ball is not rotund there will be bare points of the unit ball which are not extreme points.

In [4, Theorem 2.1] Klee showed that, if $A$ is a compact convex set in a normed linear space, then $A=\operatorname{cl}$ conv $\exp A$. Klee's proof of this result consists first, of replacing the original norm by an equivalent one 
which is rotund and smooth and second, of proving that, under the new norm, $A=\mathrm{cl}$ conv $B$ with $B$ a certain subset of $\exp A$. Klee does not define bare points as such but his set $B$ is precisely the set of bare points of $A$, under the new norm. An immediate corollary of Klee's proof is the following generalisation of Orland's and Berberian's results.

In a normed linear space in which the norm is rotund and smooth, if $A$ is a compact convex set and $B$ the set of bare points of $A$, then

$$
A=\operatorname{cl} \operatorname{conv} B \text {. }
$$

Klee's theorem also contains the assertion that $\operatorname{ext} A C \operatorname{cl} \exp A$. This is a consequence of $A=\operatorname{cl} \operatorname{conv} \exp A$ and of [3, V $\S 1$ Theorem 3]. The corresponding result for bare points ext $A C \mathrm{cl} B$ follows in the same way, if the norm is rotund and smooth.

In the finite dimensional case this result can be obtained very simply. If the space has dimension $n$ say and $M$ is any subset then conv $M$ is the set of all sums $\sum_{i=1}^{n} \lambda_{i} x_{i}$ with $x_{i} \in M, \lambda_{i} \geqq 0(i=1,2, \cdots, n)$ and $\sum_{i=1}^{n} \lambda_{i}=1$ [2, Chapter II, $\S 1$ Exercise 8].

Hence if $M$ is compact and

$$
K=\left\{\left(\lambda_{1}, \cdots, \lambda_{n}\right) \in R^{n}: \lambda_{i} \geqq 0(i=1,2, \cdots, n), \sum_{i=1}^{n} \lambda_{i}=1\right\},
$$

conv $M$ is the image of the compact set $K \times M^{n}$ under the continuous mapping

$$
\left(\lambda_{1}, \cdots, \lambda_{n}, x_{1}, \cdots, x_{n}\right) \rightarrow \sum_{i=1}^{n} \lambda_{i} x_{i} ;
$$

so that $\operatorname{conv} M$ is compact. Now if $B$ is the set of bare points of $A, \operatorname{cl} B$ is compact and hence conv $\mathrm{cl} B$ is compact and closed. It follows that $\operatorname{cl} \operatorname{conv} B=\operatorname{conv} \mathrm{cl} B$. Thus, if $p$ is an extreme point of $A, p \in \operatorname{conv} \operatorname{cl} B$ and hence, $p \in \operatorname{cl} B$.

These results raise the question of the relationship between bare points and exposed points. It is clear that an unbounded convex set has no bare points and that if the norm is not rotund there can be bare points which are not extreme points. If the norm is rotund but not smooth it is easy to produce a compact convex set having an exposed point which is not bare. Take a two dimensional subspace whose unit ball has a 'corner' and construct a closed bounded convex set which has a 'smooth' boundary through this point. The question which remains is the following. Is there a normed linear space of dimension greater than one in which, for every compact convex subset, the notions of bare point and exposed point coincide? The answer to this question is negative, as we have seen, if the norm is not both rotund and smooth. That the answer is always negative is shown by the following result. 
THEOREM. In a normed linear space of dimension greater than one there always exists a compact convex set with an exposed point which is not a baye point.

Proof. We assume, without loss of generality that the space is twodimensional and the norm is rotund and smooth. Take rectangular axes $O x, O y$ through the zero of the space and suppose a sphere of unit radius drawn to touch the $x$-axis at $O$ and to lie in the half plane $y \geqq 0$. The lower arc of this sphere will have equation $y=f(x)$ for some range of values of $x$ which we may assume to include the closed interval $[-1,1]$. The function $f$ is strictly convex, differentiable for $-1 \leqq x \leqq 1$, strictly decreasing for $-1 \leqq x \leqq 0$, and strictly increasing for $0 \leqq x \leqq 1$.

The construction which now follows was shown me by W. J. Firey. It represents a considerable simplification of the one I first used.

Define $g$ on $[-1,1]$ by

$$
g(x)=f\left(x^{2} \operatorname{sgn} x\right) \quad(-1 \leqq x \leqq 1) ;
$$

where $\operatorname{sgn} x$ is $-1,0$ or 1 according as $x$ is negative, zero or positive. (The effect of this construction is to give a function which, compared to $f$ is 'infinitely flat' near the origin). We show that $g$ is convex on $[-1,1]$. If $0 \leqq x_{1} \leqq x_{2} \leqq 1$ and $0 \leqq \lambda \leqq 1$,

$$
\begin{aligned}
g\left(\lambda x_{1}+(1-\lambda) x_{2}\right) & =f\left(\left(\lambda x_{1}+(1-\lambda) x_{2}\right)^{2}\right) \\
& \leqq f\left(\lambda x_{1}^{2}+(1-\lambda) x_{2}^{2}\right) \\
& \leqq \lambda f\left(x_{1}^{2}\right)+(1-\lambda) f\left(x_{2}^{2}\right) \\
& =\lambda g\left(x_{1}\right)+(1-\lambda) g\left(x_{2}\right) .
\end{aligned}
$$

Here we used convexity of $x^{2}$ and the fact that $f$ is increasing on $[0,1]$, then the convexity of $f$. It follows similarly that $g$ is convex on $[-1,0]$ and, because $g$ has a minimum at $x=0$, that $g$ is convex on $[-1,1]$.

Now let $A$ be the set bounded by the $\operatorname{arc} y=g(x) \quad(-1 \leqq x \leqq 1)$ and the straight line joining the ends of this arc. Because $0 \leqq g(x) \leqq f(x)$ $(-1 \leqq x \leqq 1)$ and $g(0)=0$ it is immediate that $O$ is an exposed point of $A$.

Suppose that there is a ball $D$ such that $A \subset D$ and $O$ is on the boundary sphere, $S$, of $D$. It follows that the $x$-axis is a tangent to $S$ at $O$ and from this and smoothness, that the centre of $D$ lies on the line joining $O$ to the centre of our original unit sphere. From this we deduce that, for $-1 \leqq x \leqq 1$, the equation of the lower half of $S$ is

$$
y=r f(x / r)
$$

where $r$ is the radius of $S$. If $r<1$, 


$$
\begin{aligned}
r f(1 / 2 r) & =(1-r) f(0)+r f(1 / 2 r) \\
& >f\left(\frac{1}{2}\right) \\
& >f\left(\frac{1}{4}\right) \\
& =g\left(\frac{1}{2}\right),
\end{aligned}
$$

which contradicts $A \subset D$. Thus $r \geqq 1$, and hence

$$
g(1 / 2 r)=f\left(1 / 4 r^{2}\right)<f\left(1 / 2 r^{2}\right) \leqq r f\left(1 / 2 r^{2}\right)
$$

which also contradicts $A \subset D$. It follows that $O$ is not a bare point of $A$, and our theorem is proved.

We close with the remark that if we can assume that $f$ has a positive second derivative at the origin a much easier counterexample can be constructed using the function $e^{-1 / x^{2}}$.

\section{References}

[1] S. K. Berberian, 'Compact convex sets in inner product spaces', American Math. Monthly 74 (1967), 702-704.

[2] N. Bourbaki, Espaces vectoriels topologiques, Actualités Scientifiques et Industrielles 1189, (Hermann, Paris, 1953).

[3] M. M. Day, Normed linear spaces (second printing, Springer-Verlag, Berlin-GöttingenHeidelberg, 1962).

[4] V. L. Klee jr. 'Extremal structure of convex sets. II, Math. Z. 69 (1958), 90-104.

[5] G. H. Orland, 'On a class of operators', Proc. American Math. Soc. 15 (1964), 75-79.

University of Otago

Dunedin, New Zealand 